Korean J. Math. 22 (2014), No. 1, pp. 29-36

http://dx.doi.org/10.11568/kjm.2014.22.1.29

\title{
ON THE MARTINGALE EXTENSION OF LIMITING DIFFUSION IN POPULATION GENETICS
}

\author{
WON CHOI
}

\begin{abstract}
The limiting diffusion of special diploid model can be defined as a discrete generator for the rescaled Markov chain. Choi( [2] ) defined the operator of projection $S_{t}$ on limiting diffusion and new measure $d Q=S_{t} d P$. and showed the martingale property on this operator and measure. Let $P_{\rho}$ be the unique solution of the martingale problem for $\mathcal{L}_{0}$ starting at $\rho$ and $\pi_{1}, \pi_{2}, \cdots, \pi_{n}$ the projection of $E^{n}$ on $x_{1}, x_{2}, \cdots, x_{n}$. In this note we define

$$
d Q_{\rho}=S_{t} d P_{\rho}
$$
\end{abstract}

and show that $Q_{\rho}$ solves the martingale problem for $\mathcal{L}_{\pi}$ starting at $\rho$.

\section{Introduction}

Let $E$ (a locally compact separable metric space) be the set of all possible allels and $\nu_{0}($ in $\mathcal{P}(E)$, the set of Borel probability measures on $E$ ) the distribution of the type of a new mutant. Suppose that $N($ a positive integer) is the diploid population size and $s(\mathbf{x})$ is the selection coefficient of allele $\mathbf{x}$.

Received July 26, 2013. Revised September 28, 2013. Accepted September 28, 2013.

2010 Mathematics Subject Classification: 92D10, 60H30, 60G44.

Key words and phrases: Diploid model, Limiting diffusion, Martingale problem, Population genetics.

This research was supported by Incheon National University Research Grant, 2013-2014.

(c) The Kangwon-Kyungki Mathematical Society, 2014.

This is an Open Access article distributed under the terms of the Creative commons Attribution Non-Commercial License (http://creativecommons.org/licenses/by -nc/3.0/) which permits unrestricted non-commercial use, distribution and reproduction in any medium, provided the original work is properly cited. 
We now consider the normal-selection model which define in W. Choi( [1]). The type space $E$ is unspecified. However, $\nu_{0}$ and the function $s$ must jointly satisfy the following condition; If $X$ is a random variable with distribution $\nu_{0}$, then $s(X)$ has the normal distribution with mean 0 and variance $\sigma^{2}$. Furthermore, $\sigma=\sigma_{0} / 2 N$ for an appropriate constant $\sigma_{0}$. There are therefore a number of possible choice for $E, \nu_{0}$, and $s$, including;

$$
E=(0,1), \nu_{0}=U(0,1), s(\mathbf{x})=\sigma \boldsymbol{\Phi}^{-1}(\mathbf{x}),
$$

where $\Phi$ is the standard normal distribution function,

$$
E=R, \nu_{0}=N\left(0, \sigma^{2}\right), s(\mathbf{x})=\mathbf{x},
$$

and

$$
E=R, \nu_{0}=N\left(0, \sigma_{0}^{2}\right), s(\mathbf{x})=\mathbf{x} / 2 N .
$$

For each positive integer $M$, let $\omega_{M}$ be a positive, symmetric, bounded, Borel function on $E^{2}$, let $R_{M}((p, q), d x \times d y)$ be an one-step transition function on $E^{2} \times \mathcal{B}\left(E^{2}\right)$ satisfying

$$
R_{M}((p, q), d x \times d y)=R_{M}((q, p), d y \times d x),
$$

and $Q_{M}(p, d x)$ be an one-step transition function on $E \times \mathcal{B}(E)$.

Let $N$ be the diploid population size. We consider $M=2 N$ gametes and the mapping $\eta_{M}: E^{M} \rightarrow \mathcal{P}(E)$ by letting

$$
\eta_{M}\left(p_{1}, p_{2}, \cdots, p_{M}\right)=\frac{1}{M}\left(\delta_{p_{1}}+\delta_{p_{2}}+\cdots+\delta_{p_{M}}\right) .
$$

Here $\delta_{p} \in \mathcal{P}(E)$ denotes the unit mass at $p \in E$. The state space for this model is

$$
\mathcal{K}_{M}(E)=\eta_{M}\left(E^{M}\right) .
$$

Given $\mu \in \mathcal{P}(E)$, we define $\mu_{1} \in \mathcal{P}\left(E^{2}\right)$ and $\mu_{2}, \mu_{3} \in \mathcal{P}(E)$ by

$$
\begin{aligned}
\mu_{1}(d p \times d q) & =\omega_{M}(p, q) \mu^{2}(d p \times d q) /\left\langle\omega_{M}, \mu^{2}\right\rangle, \\
\mu_{2}(d x) & =\int_{E^{2}} R_{M}((p, q), d x \times E) \mu_{1}(d p \times d q), \\
\mu_{3}(d x) & =\int_{E} Q_{M}(p, d x) \mu_{2}(d p) .
\end{aligned}
$$

The Markov chain has one-step transition function $P_{M}(\mu, d \theta)$ on $\mathcal{K}_{M}(E) \times$ $\left(\mathcal{K}_{M}(E)\right)$ defined by

$$
P_{M}(\mu, \cdot)=\int_{E^{M}}\left(\mu_{3}\right)^{M}\left(d p_{1} \times d p_{2} \times \cdots \times d p_{M}\right) \delta_{\eta_{M}\left(p_{1}, p_{2}, \cdots, p_{M}\right)}(\cdot) .
$$


Choi( [1]) identified and characterized the limiting diffusion of this diploid model by defining discrete generator for the rescaled Markov chain. Also he defined the operator of projection $S_{t}$ on limiting diffusion and new measure $d Q=S_{t} d P$, and showed the martingale property on this operator and measure. ( [2]) Let $P_{\rho}$ be the unique solution of the martingale problem for $\mathcal{L}_{0}$ starting at $\rho$ and $\pi_{1}, \pi_{2}, \cdots, \pi_{n}$ the projections of $E^{n}$ on $x_{1}, x_{2}, \cdots, x_{n}$. In this note we define

$$
d Q_{\rho}=S_{t} d P_{\rho}
$$

and show that $Q_{\rho}$ solves the martingale problem for $\mathcal{L}_{\pi}$ starting at $\rho$.

\section{Main Results}

We define the discrete generator $\mathcal{L}_{M}$ for the $M$-the rescaled Markov chain and canonical coordinate process $\left\{\rho_{t}, t \geq 0\right\}$ :

$$
\left(\mathcal{L}_{M} \phi\right)\left(\rho_{t}\right)=M \int_{\mathcal{P}_{M}}\left(\phi\left(\nu_{t}\right)-\phi\left(\rho_{t}\right)\right) P_{M}\left(\rho_{t}, \nu_{t}\right)
$$

where $\mathcal{P}_{M}$ is given in the diploid models as described above.

We restrict our attention to test functions $\theta$ of the form

$$
\theta\left(\nu_{t}\right)=\beta_{1}\left\langle f_{1}, \nu_{t}\right\rangle \cdots \beta_{k}\left\langle f_{k}, \nu_{t}\right\rangle, \quad \theta\left(\rho_{t}\right)=\left\langle f_{1}, \rho_{t}\right\rangle \cdots\left\langle f_{k}, \rho_{t}\right\rangle
$$

where $f_{1}, \cdots, f_{k} \in \mathcal{B}(E)$ and $\left\{\beta_{i}\right\}$ is a set of non-negative constants satisfying that $\sup _{i} \beta_{i}<+\infty$. Assume that "mutation or gene conversion rate" is

$$
\sum_{k \in S} \beta_{k}\left\langle f_{i}, \rho_{t}\right\rangle-\beta_{i}-\beta_{j} \text { for every } i<j,
$$

in the diploid models as described above. This means the mutations or gene conversions occur with particular rate in case of $i<j$. See [3].

We start with;

Lemma 1. Suppose that there exist a selection function $\sigma$ on $E^{2}$ and bounded linear operator $A, B$ on $\mathcal{B}(E)$ such that

$$
\begin{aligned}
\omega_{M}(p, q) & =1+\frac{1}{M} \sigma(p, q)+o\left(\frac{1}{M}\right), \\
\int_{S} f(x) R_{M}((p, q), d x \times S) & =f(p)+\frac{1}{M}(B f)(p, q)+o\left(\frac{1}{M}\right), \\
\int_{S} f(x) Q_{M}(p, d x) & =f(x)+\frac{1}{M}(A f)(p)+o\left(\frac{1}{M}\right) .
\end{aligned}
$$


Then there exist $a_{f_{i}, f_{j}}, b_{f_{i}} \in \mathcal{B}(\mathcal{P}(E))$ such that

$$
\begin{aligned}
\lim _{M \rightarrow \infty}\left(\mathcal{L}_{M} \theta\right)\left(\rho_{t}\right)=\left(\mathcal{L}_{\pi} \theta\right)\left(\rho_{t}\right)= & \\
& \sum_{1 \leq i<j \leq k} a_{f_{i}, f_{j}} F_{z_{i} z_{j}}\left(\left\langle\mathbf{f}, \rho_{t}\right\rangle\right)+\sum_{i=1}^{k} b_{f_{i}} F_{z_{i}}\left(\left\langle\mathbf{f}, \rho_{t}\right\rangle\right)
\end{aligned}
$$

uniformly in $\rho_{t} \in \mathcal{K}_{M}(E)$, where $F_{z_{i}}$ and $F_{z_{i} z_{j}}$ mean the partial derivative with respect to $i$ and $i, j$, respectively. Here

$$
\begin{aligned}
\theta\left(\rho_{t}\right) & =F\left(\left\langle f_{1}, \rho_{t}\right\rangle,\left\langle f_{2}, \rho_{t}\right\rangle, \cdots,\left\langle f_{k}, \rho_{t}\right\rangle\right)=F\left(\left\langle\mathbf{f}, \rho_{t}\right\rangle\right) \\
a_{f_{i}, f_{j}} & =\beta_{i}\left\langle f_{i} f_{j}, \rho_{t}\right\rangle-\left\langle f_{i}, \rho_{t}\right\rangle\left\langle f_{j}, \rho_{t}\right\rangle\left(\sum_{k \in S} \beta_{k}\left\langle f_{i}, \rho_{t}\right\rangle-\beta_{i}-\beta_{j}\right) \\
b_{f_{i}} & =\left\langle A f_{i}, \rho_{t}\right\rangle+\left\langle B f_{i}, \rho_{t}^{2}\right\rangle+\left\langle\left(f_{i} \circ \pi\right) \sigma, \rho_{t}^{2}\right\rangle-\left\langle f_{i}, \rho_{t}\right\rangle\left\langle\sigma, \rho_{t}^{2}\right\rangle,
\end{aligned}
$$

and $\pi$ is the projection of $E^{2}$.

Proof. See [1].

In particular, the set of possible alleles, known as the type space, is a locally compact, separable metric space $E$ and the mutation operator $A$ is given

where $\theta>0$.

$$
A f=\frac{1}{2} \theta\left(\left\langle f, \nu_{0}\right\rangle-f\right)
$$

Let $\pi_{1}, \pi_{2}, \cdots, \pi_{n}$ be the projection of $E^{n}$ on $x_{1}, x_{2}, \cdots, x_{n}$-coordinate, respectively. Define

$$
S_{t}^{\pi_{x}, \pi_{x}+\pi_{y}}=\exp \left\{\left\langle\pi_{y}, \rho_{t}\right\rangle-\left\langle\pi_{y}, \rho_{0}\right\rangle-\int_{0}^{t} e^{-\left\langle\pi_{y} \cdot \rho_{s}\right\rangle} \mathcal{L}_{\pi_{x}} e^{\left\langle\pi_{y}, \rho_{s}\right\rangle} d s\right\}
$$

where $x, y=1,2, \cdots, n, x \neq y$. For $\rho$, we denote by $P_{\rho}$ the unique solution of the martingale problem for $\mathcal{L}_{0}$ (i.e., the distribution of the neutral model) starting at $\rho_{0}$.

Theorem 2 allows us to define a mean one $\left\{\mathcal{F}_{t}\right\}$-martingale.

Theorem 2. Suppose $\left\{\mathcal{F}_{t}\right\}$ is corresponding filtration with respect to topology of uniform convergence on compact sets. For $0<\delta<\delta_{0}$, there exists $\delta_{0}$ such that

$$
E^{P_{\rho}}\left[\frac{S_{t+\delta}^{0, \pi}}{S_{t}^{0, \pi} \mid \mathcal{F}_{t}}\right]=1
$$


Proof. Define $\pi_{K}=(-K) \vee(\pi \wedge K), K=1,2, \cdots, n$ and note that $\left\{S_{t}^{0, \pi_{K}}\right\}$ is mean-one martingale from [2]. Hence

$$
E^{P_{\rho}}\left[\frac{S_{t+\delta}^{0, \pi_{K}}}{S_{t}^{0, \pi_{K}}} e^{\left\langle\pi_{K}, \rho_{t}\right\rangle+\frac{1}{2} \theta \delta\left\langle\pi_{K}, \nu_{0}\right\rangle} \mid \mathcal{F}_{t}\right]=e^{\left\langle\pi_{K}, \rho_{t}\right\rangle+\frac{1}{2} \theta \delta\left\langle\pi_{K}, \nu_{0}\right\rangle}
$$

But,

$$
\begin{aligned}
\exp \left(\left\langle\pi_{K}, \rho_{t}\right\rangle+\frac{1}{2} \theta \delta\left\langle\pi_{K}, \nu_{0}\right\rangle\right) & \leq \exp \left(\left\langle\pi_{K}, \rho_{t}\right\rangle+\frac{1}{2} \theta \int_{t}^{t+\delta}\left\langle\pi_{K}, \rho_{s}\right\rangle d s\right) \\
& \leq \exp \left(\left\langle\pi_{1}, \rho_{t+\delta}\right\rangle+\frac{1}{2} \theta \int_{t}^{t+\delta}\left\langle\pi_{1}, \rho_{s}\right\rangle d s\right)
\end{aligned}
$$

We apply the dominated convergence theorem to show that the right hand side of above inequality is integrable. Let $q=\frac{p}{p-1}$ and define $\delta_{0}=2 p / \theta q$. By the Hölder and Jensen inequalities,

$$
\begin{aligned}
E^{P_{\rho}}[ & \left.\exp \left(\left\langle\pi_{1}, \rho_{t+\delta}\right\rangle+\frac{1}{2} \theta \int_{t}^{t+\delta}\left\langle\pi_{1}, \rho_{s}\right\rangle d s\right)\right] \\
& \leq\left(E^{P_{\rho}}\left[\exp \left(p\left\langle\pi_{1}, \rho_{t+\delta}\right\rangle\right)\right]\right)^{1 / p}\left(E^{P_{\rho}}\left[\exp \left(\frac{1}{2} \theta q \int_{t}^{t+\delta}\left\langle\pi_{1}, \rho_{s}\right\rangle d s\right)\right]\right)^{1 / q} \\
& \leq E^{P_{\rho}}\left[\left\langle e^{p \pi_{1}}, \rho_{t+\delta}\right\rangle\right]^{1 / p}\left(\frac{1}{\delta} \int_{t}^{t+\delta} E^{P_{\rho}}\left[\left\langle e^{\theta q \delta \pi_{1} / 2}, \rho_{s}\right\rangle\right] d s\right)^{1 / q}
\end{aligned}
$$

Let $\{U(t)\}$ be the semigroup on $\mathcal{B}(E)$ with generator $A$. Since

$$
E^{P_{\rho}}\left[\left\langle g, \rho_{t}\right\rangle\right]=\langle U(t) g, \rho\rangle \leq\langle g, \rho\rangle \vee\left\langle g, \nu_{0}\right\rangle
$$

for $g \in \mathcal{B}(E)$, we have

$$
\begin{aligned}
E^{P_{\rho}} & \left.\exp \left(\left\langle\pi_{1}, \rho_{t+\delta}\right\rangle+\frac{1}{2} \theta \int_{t}^{t+\delta}\left\langle\pi_{1}, \rho_{s}\right\rangle d s\right)\right] \\
& \leq\left[\left\langle e^{p \pi_{1}}, \rho\right\rangle \vee\left\langle e^{p \pi_{1}}, \nu_{0}\right\rangle\right]^{1 / p}\left[\left\langle e^{\theta q \delta \pi_{1} / 2}, \rho\right\rangle \vee\left\langle e^{\theta q \delta \pi_{1} / 2}, \nu_{0}\right\rangle\right]^{1 / q} \\
& \leq\left\langle e^{p \pi_{1}}, \rho\right\rangle \vee\left\langle e^{p \pi_{1}}, \nu_{0}\right\rangle
\end{aligned}
$$

if $0<\delta<\delta_{0}$, and the proof is complete.

Theorem 2 allows us to define $Q_{\rho}$ by

$$
d Q_{\rho}=S_{t}^{0, \pi} d P_{\rho} .
$$


Choi( [2]) proved that $Q_{\rho}^{K}$ solves the martingale problem for $\mathcal{L}_{\pi_{K}}$ starting at $\rho_{0}$. In advance, we now show that $Q_{\rho}$ solve the martingale problem for $\mathcal{L}_{\pi}$ starting at $\rho_{0}$.

Theorem 3. The measure $Q_{\rho}$ is a solution of the $\left(E^{n}, \mathcal{L}_{\pi}, \rho_{0}\right)$ martingale problem.

Proof. Define

$$
M_{t}^{\pi}=\phi\left(\rho_{t}\right)-\phi\left(\rho_{0}\right)-\int_{0}^{t}\left(\mathcal{L}_{\pi} \phi\right)\left(\rho_{s}\right) d s
$$

and

$$
M_{t}^{\pi_{K}}=\phi\left(\rho_{t}\right)-\phi\left(\rho_{0}\right)-\int_{0}^{t}\left(\mathcal{L}_{\pi_{K}} \phi\right)\left(\rho_{s}\right) d s
$$

for $\phi \in \mathcal{D}\left(\mathcal{L}_{\pi}\right)$. Then $M_{t}^{\pi_{K}}$ is $Q_{\rho}^{K}$-martingale for $Q_{\rho}^{K}$ from the result of [2] with $\pi$ replaced by $\pi_{K}$ and we have

$$
E^{Q_{\rho}^{K}}\left[M_{t+\delta}^{\pi_{K}}-M_{t}^{\pi_{K}} \mid \mathcal{F}_{t}\right]=0
$$

Hence

$$
E^{P_{\rho}}\left[\left(M_{t+\delta}^{\pi_{K}}-M_{t}^{\pi_{K}}\right) S_{t+\delta}^{0, \pi_{K}} \mid \mathcal{F}_{t}\right]=0
$$

and

$$
E^{P_{\rho}}\left[\left(M_{t+\delta}^{\pi_{K}}-M_{t}^{\pi_{K}}\right) \frac{S_{t+\delta}^{0, \pi_{K}}}{S_{t}^{0, \pi_{K}}} e^{\left\langle\pi_{K}, \rho_{t}\right\rangle+\frac{1}{2} \theta \delta\left\langle\pi_{K}, \nu_{0}\right\rangle} \mid \mathcal{F}_{t}\right]=0 .
$$

Note that the integrand in above equation is bounded by the argument used for Theorem 2. For such $\delta$ used at Theorem 2, we conclude that

$$
E^{P_{\rho}}\left[\left(M_{t+\delta}^{\pi}-M_{t}^{\pi}\right) \frac{S_{t+\delta}^{0, \pi}}{S_{t}^{0, \pi}} e^{\left\langle\pi, \rho_{t}\right\rangle+\frac{1}{2} \theta \delta\left\langle\pi, \nu_{0}\right\rangle} \mid \mathcal{F}_{t}\right]=0 .
$$

On the other hand,

$$
E^{P_{\rho}}\left[\left(M_{t}^{\pi_{K}}\right)^{2} S_{t}^{0, \pi_{K}}\right]=E^{Q_{\rho}^{K}}\left[\left(M_{t}^{\pi_{K}}\right)^{2}\right]=E^{Q_{\rho}^{K}}\left[\left\langle\left\langle M \pi_{K}\right\rangle\right\rangle_{t}\right] \leq C t
$$

where $\left\langle\left\langle M \pi_{K}\right\rangle\right\rangle_{t}=\int_{0}^{t} \psi\left(\pi_{s}\right) d s$ is increasing process and $C$ is a constant with

$$
\begin{aligned}
& \psi\left(\pi_{s}\right)= \\
& \sum_{i, j=1}^{k}\left(\beta_{i}\left\langle f_{i} f_{j}, \rho_{t}\right\rangle-\left\langle f_{i}, \rho_{t}\right\rangle\left\langle f_{j}, \rho_{t}\right\rangle\left(\sum_{k} \beta_{k}\left\langle f_{i}, \rho_{t}\right\rangle-\beta_{i}-\beta_{j}\right)\right) F_{z_{i} z_{j}}\left(\left\langle\mathbf{f}, \rho_{t}\right\rangle\right) \leq C .
\end{aligned}
$$


From the Fatou's lemma, we have

$$
E^{P_{\rho}}\left[\left(M_{t}^{\pi}\right)^{2} S_{t}^{0, \pi}\right] \leq C t
$$

and

$$
E^{Q_{\rho}}\left[\left(M_{t}^{\pi}\right)^{2}\right] \leq C t .
$$

Therefore the integrands in below equations are integrable and we conclude that

$$
E^{P_{\rho}}\left[\left(M_{t+\delta}^{\pi}-M_{t}^{\pi}\right) S_{t+\delta}^{0, \pi} \mid \mathcal{F}_{t}\right]=0
$$

and

$$
E^{Q_{\rho}^{K}}\left[M_{t+\delta}^{\pi}-M_{t}^{\pi} \mid \mathcal{F}_{t}\right]=0
$$

By Theorem 3, we know that there exists a probability measure $Q_{\rho}$ satisfying the following conditions ;

(1) $Q_{\rho}\left(\rho(0)=\rho_{0}\right)=1$ and

(2) denoting $M_{\phi_{1}}(t)=\phi_{1}(\rho(t))-\int_{0}^{t} \mathcal{L}_{\pi} \phi_{1}(\rho(s)) d s, M_{\phi_{1}}(t)$ is a $Q_{\rho^{-}}$ martingale.

Therefore we conclude with;

Corollary 4. Defining

$$
\begin{gathered}
\left\langle\phi_{1}, \phi_{2}\right\rangle \equiv \mathcal{L}_{\pi}\left(\phi_{1} \cdot \phi_{2}\right)-\phi_{1} \mathcal{L}_{\pi} \phi_{2}-\phi_{2} \mathcal{L}_{\pi} \phi_{1} \\
\left(M_{\phi_{1}}(t)\right)^{2}-\int_{0}^{t}\left\langle\phi_{1}, \phi_{2}\right\rangle(\rho(s)) d s
\end{gathered}
$$

is a $Q_{\rho}$-martingale.

Proof. Since the measure $Q_{\rho}$ is a solution of $L_{\pi}$-martingale problem, the result directly follows from quadratic covariation process.

\section{References}

[1] W.Choi, On the limiting diffusion of special diploid model in population genetics, Bull. Korean Math. Soc. 42 (2) (2005), 397-404.

[2] W.Choi, On the martingale property of limiting diffusion in special diploid model, J. Appl. Math. info. 31 (1) (2013), 241-246.

[3] A.Shimizu, Stationary distribution of a diffusion process taking values in probability distributions on the partitions, Proceedings of a Workshop held in Nagoya, (1985), 100-114. 
Won Choi

Department of Mathematics

Incheon National University

Incheon 406-772, Korea

E-mail: choiwon@incheon.ac.kr 